THEORY AND METHODS

\title{
Care Need Index, a useful tool for the distribution of primary health care resources
}

\author{
K Sundquist, M Malmström, S-E Johansson, J Sundquist
}

J Epidemiol Community Health 2003;57:347-352

See end of article for authors' affiliations

\section{Correspondence to}

Dr K Sundquist, Family Medicine Stockholm,

Karolinska Institutet, Alfred

Nobels allé 12, SE-14183

Huddinge, Sweden;

Kristina.Marinko@

klinvet.ki.se

Accepted for publication

15 September 2002

\begin{abstract}
Study objective: To demonstrate how Care Need Index (CNI), a social deprivation index, may be used to allocate total primary health care resources.

Design: Cross sectional survey and register data. The $\mathrm{CNI}$ was based on sociodemographic factors: elderly persons living alone, children under age 5, unemployed people, people with low educational status, single parents, high mobility, and foreign born people. The CNI weights were calculated from the ratings of Swedish GPs of the impact of these factors on their workload. The CNI scale was transformed into a positive scale to avoid negative values. $\mathrm{CNI}$ weights were calculated for each decile of the study population. The risk of poor self reported health in the $\mathrm{CNI}$ deciles was estimated by means of a hierarchical logistic regression in the age range 25-74 ( $n=27346)$. The MigMed database comprising all people living in Sweden was used to calculate the CNI for Stockholm.

Participants: The Swedish population and the population in Stockholm County.

Main results: The means of the CNI for deciles ranged from 61 (most affluent neighbourhoods) to 140 (most deprived) in Stockholm County. The ratio between the tenth and the first decile was 1.66. There was an approximately $150 \%$ increased risk of poor self reported health for people living in the most disadvantaged neighbourhoods $(O R=2.50)$ compared with those living in the most affluent ones $(O R=1)$. CNI ratios for the deciles corresponded approximately to the odds ratios of poor self reported health status.
\end{abstract}

Conclusions: The $\mathrm{CNI}$ can be used to allocate total primary health care resources.
$\mathrm{T}$ he purpose of this study is to show how a modified Jarman score, such as the Care Need Index $(\mathrm{CNI}),{ }^{1}$ can be used as a direct empirical measure for the distribution of primary health care resources. Existing indices such as the Jarman score ${ }^{2}$ allocate an additional fee, above a certain cut off, to general practitioners (GPs) who have their practices in the $5 \%$ most deprived neighbourhoods. However, the presence of negative values in the scores makes it difficult to use them for a total distribution of resources. This study shows how such an index can be transformed into a more useful scale in order to allocate total resources to primary health care.

The CNI was originally developed for the purpose of measuring the potential workload of GPs in Swedish primary health care using indicators for material deprivation (unskilled, unemployed, and living in crowded households) and adding demographic factors related to family structures (elderly persons living alone, children under age 5 , and single parent families), social instability (people who had moved house during the past year), and cultural needs (ethnicity) weighted by Swedish GPs. ${ }^{1}$

Scandinavian studies showed an ecological association between deprived neighbourhoods measured by CNI and indicators of poor health, such as increased admissions to psychiatric departments, ${ }^{3-5}$ a greater number of emergency room visits, ${ }^{67}$ high CHD mortality, ${ }^{8}$ and higher consultation rates for primary health care.'

In Stockholm County, which represents one fifth of the Swedish population, a population based resource allocation model based on consumption and need is used to distribute funds for purchasing health care services from providers of primary health care. ${ }^{10}$ In this paper we propose a new model, based on measures of material and social need for medical service summed up to form an index, for the distribution of primary health care resources.

The first aim of this study is to transform the CNI into a positive scale for allocating primary health care resources totally, not only to the most deprived areas. The second aim is to compare the means of the CNI (CNI weights) categorised into deciles, from the most affluent neighbourhoods (lst decile) to the most deprived ones (10th decile), with the risk of poor self reported health status in every decile. The third aim is to exemplify how primary health care resources in Stockholm County ( 1.7 million inhabitants) can be allocated by the transformed CNI, compared with the official consumption model.

\section{METHODS}

Data sources 1: A sample of the Swedish population

The aim was to analyse the relation between the transformed CNI and poor self reported health in deciles of a sample of the Swedish population. The sample consists of individual data, comprising face to face interviews, from the Swedish Annual Level of Living Survey (SALLS), matched with the social position of the area (small area market statistics, SAMS) in which the respondents live, the latter being measured by CNI. ${ }^{1}$ This study is based on pooled data from $1992-97^{11}$ for the age range 25-74 and consists of 27346 interviews. The CNI scores could not be matched with SAMS data for 1294 persons, representing about $4.7 \%$ of all participants. The ethics committee at Huddinge University Hospital, Stockholm, has approved this study.

The non-response rate for SALLS was about $20 \%$. Refusal was the predominant reason for not responding (more than two thirds of the non-responses consisted of refusals). One fifth of all non-respondents were not found, and one tenth were ill. Mortality as a measure of ill health among both

Abbreviations: CNI, Care Need Index; SALLS, Swedish Annual Level of Living Survey; SAMS, small area market statistics 
Table 1 The transformation of the CNI, a comparison

\begin{tabular}{llll}
\hline & Original CNI & CNI & Transformed CNI \\
\hline Variables & Elderly living alone & Elderly living alone & Elderly living alone \\
& Children under age 5 & Children under age 5 & Children under age 5 \\
& Unemployed people & Unemployed people & Unemployed people \\
& Unskilled workers & Low level education & Low level education \\
& Single parents & Single parents & Single parents \\
& High mobility & High mobility & High mobility \\
& Foreign born people & Foreign born people & Foreign born people \\
& Overcrowding & & \\
Range & $-76-+54$ & $-54-+78$ & $50-164$ \\
Mean & 0 & 0 & 100 \\
SD & 15 & 16.7 & 15 \\
\hline
\end{tabular}

respondents and non-respondents was analysed in a proportional hazard model adjusted for sex, age, marital status, and geographical area, using the data supplied by the SALLS samples. It was found that those who refused to participate had the same mortality rate as the respondents, while the other two groups (that is, those who could not be found and those who were ill) had significantly higher mortality.

\section{Outcome variable}

Poor self reported health status is based on the question: "How would you describe your general health?" There were three response alternatives: good, bad, or anywhere between good and bad. Those who answered that their health status was bad or anywhere between good and bad were counted as having a poor self reported health status.

\section{Independent variables}

Individual level

Age (continuous) and sex

\section{Second level \\ CNI calculated for SAMS}

SAMS refers to neighbourhoods with an average population of about 2000 for Stockholm, and about 1000 for the rest of Sweden (total, 9667). The boundaries of SAMS neighbourhoods are drawn to include similar types of housing construction.

\section{Data sources 2: MigMed databases}

To calculate the CNI for all people living in Stockholm County, we used the MigMed database at the Karolinska Institutet, Sweden. The database includes the whole Swedish population. In this study 1.7 million people, the total population of Stockholm County, were used for the analysis. The database contains data on sociodemographic factors, including age, gender, socioeconomic status (SES) (education, income), country of birth, and area (9677 neighbourhoods, boundaries defined by housing structure for administrative and commercial purposes). MigMed is linked to several other databases: Louise: A register including the entire population. The database contains annual specifications of: income, education, place of residence (geographical coordinates). The Immigration Register contains data on country of birth. The Total Population Register (TPR) comprises all people who have a residence permit. People who stay more than six months in Sweden will have a permit and are included in the TPR. It includes data on mobility from one neighbourhood to another, out migration and any instances of return to Sweden. The undercoverage and overcoverage is very low.

\section{CNI: calculation and transformation of $\mathrm{CNI}$ into a positive scale}

Neighbourhood social position, measured by the CNI, was calculated for all SAMS neighbourhoods except those with less than 50 inhabitants (in total 943), which were excluded because of the instability of the estimated proportions.

The CNI included proportions of seven material, sociodemographic, and cultural variables from Swedish public registers for each SAMS area: elderly people living alone, children under age 5, unemployed people, people with low educational status, single parents, residents who have moved house during the past year, and foreign born people from Southern and Eastern Europe, Asia, Africa, and South America (table 1).

In the autumn of 2000, all 3922 Swedish GPs received a questionnaire that 2541 answered (response rate $64.8 \%$ ), rating the impact of these factors on their workload. The CNI was based on these weights. The proportions of the variables were normalised by applying an angular transformation. The means and standard deviations of the proportions were calculated for Sweden as a whole. For each area, all seven proportions were standardised by calculating Z scores. ${ }^{1}$ The standardised value of each variable was multiplied by the corresponding new weights based on the survey of the Swedish GPs. The mean value of CNI for the whole of Sweden was 0 with $\mathrm{SD}=16.7$. The higher the index value, the more deprived was the neighbourhood. The CNI scores for Sweden ranged from -54 (most affluent neighbourhood) to 78 (most deprived neighbourhood) (table 1). The CNI scale for Sweden was transformed into a positive scale $($ mean $=100 ; \mathrm{SD}=15)$ to avoid negative values according to the formula: $Y_{j}=\left(S_{y} / S_{x}\right) \times$ $\left(X_{j}-M_{x}\right)+M_{y}$

Where:

$\mathrm{Y}_{\mathrm{j}}$ is the individual $\mathrm{j}$ value in the transformed distribution,

$S_{v}$ is the standard deviation in the transformed distribution (16.7),

$M_{y}$ is the mean value in the transformed distribution (100),

$\mathrm{X}_{\mathrm{j}}$ is the individual $\mathrm{j}$ value in the original distribution,

$\mathrm{S}_{\mathrm{x}}$ is the standard deviation in the original distribution (15),

$M_{x}$ is the mean value in the original distribution (0).

\section{Allocation of resources by CNI: The Stockholm County example}

The CNI was used to measure the social position of the area. In our MigMed database, each person in Stockholm County has an area social position measured by the CNI according to their area of living. The whole population of Stockholm County was divided into deciles according to each peson's area social position with CNI values ranging from 61 in the most affluent decile to 140 in the most deprived decile. Each decile comprised approximately 170000 people. In the next step, the means of the transformed CNI for Stockholm County were calculated for each decile. These means were used as CNI weights. These CNI weights for each decile of the population of Stockholm County were used in the calculation of new CNI weights for six administrative areas in Stockholm County.

Stockholm County is divided into six independent administrative areas: north east (NE), north west (NW), northern 
Table 2 The risk (odds ratio (OR) with 95\% confidence intervals (CI)) and prevalence $(\%)$ of poor self reported health in different $\mathrm{CNI}$ deciles in a model adjusted for sex and age (25-74 years), analysed by means of a hierarchical logistic model, based on the Swedish Annual Level of Living Survey 1992-97 (all Sweden); $\mathrm{n}=27346 . \mathrm{N}$ is the estimated population and $\mathrm{n}$ the sample size

\begin{tabular}{llllll}
\hline CNI deciles for Sweden & OR & $95 \%$ Cl & Prevalence & $n$ & $\mathrm{~N}$ \\
\hline $1(50.1-<83.0)$ & 1 & Reference & 18.0 & 2607 & 503000 \\
$2(83.0-<88.2)$ & 1.17 & 0.98 to 1.38 & 19.6 & 2619 & 503000 \\
$3(88.2-<91.7)$ & 1.30 & 1.10 to 1.54 & 21.4 & 2594 & 488000 \\
$4(91.7-<94.9)$ & 1.48 & 1.25 to 1.75 & 23.8 & 2601 & 493000 \\
$5(94.9-<98.0)$ & 1.39 & 1.18 to 1.65 & 22.8 & 2610 & 494000 \\
$6(98.0-<101.5)$ & 1.55 & 1.31 to 1.84 & 24.9 & 2601 & 497000 \\
$7(101.5-<105.2)$ & 1.54 & 1.30 to 1.82 & 24.8 & 2610 & 498000 \\
$8(105.2-<110.6)$ & 1.77 & 1.50 to 2.09 & 27.3 & 2600 & 501000 \\
$9(110.6-<120.3)$ & 1.74 & 1.47 to 2.06 & 27.2 & 2605 & 509000 \\
$10(\geqslant 120.3)$ & 2.50 & 2.12 to 2.95 & 33.8 & 2605 & 514000 \\
Missing & 1.51 & 1.25 to 1.81 & 24.1 & 1294 & 243000 \\
\hline
\end{tabular}

Table 3 Weights and ratios (weight divided by 78) for the transformed CNI scale for Sweden and Stockholm County by CNI decile and with about 170000 persons in each decile (Stockholm County)

\begin{tabular}{lllll}
\hline $\begin{array}{l}\text { CNI deciles for } \\
\text { Sweden }\end{array}$ & $\begin{array}{l}\text { Mean CNI weight } \\
\text { for Sweden } \\
\text { (transformed) }\end{array}$ & CNI ratio Sweden & $\begin{array}{l}\text { Mean CNI weight } \\
\text { Stockholm County } \\
\text { (transformed) }\end{array}$ & $\begin{array}{l}\text { CNI ratio } \\
\text { Stockholm County }\end{array}$ \\
\hline $1(50.1-<83.0)$ & 78 & 1 & 78 & 1 \\
$2(83.0-<88.2)$ & 86 & 1.11 & 84 & 1.08 \\
$3(88.2-<91.7)$ & 90 & 1.16 & 89 & 1.14 \\
$4(91.7-<94.9)$ & 93 & 1.20 & 93 & 1.19 \\
$5(94.9-<98.0)$ & 96 & 1.24 & 97 & 1.24 \\
$6(98.0-<101.5)$ & 100 & 1.28 & 100 & 1.29 \\
$7(101.5-<105.2)$ & 103 & 1.33 & 104 & 1.33 \\
$8(105.2-<110.6)$ & 108 & 1.38 & 110 & 1.50 \\
$9(110.6-<120.3)$ & 115 & 1.48 & 117 & 1.66 \\
$10(\geqslant 120.3)$ & 132 & 1.70 & 130 & \\
\hline
\end{tabular}

Stockholm (NS), southern Stockholm (SS), south west (SW), and south east (SE).

For the respective administrative area the following calculation was made:

(1) the CNI weights were multiplied by the size of the population $(\mathrm{N})$ of each of the CNI deciles so that 10 values $(\mathrm{CNI}$ weights $\times \mathrm{N})$ were obtained.

(2) These 10 values were summarised into one sum [ $\Sigma_{\text {decile }}$ 1-decile 10 (CNI weights $\mathrm{x} N$ )].

(3) The CNI weights for each decile were summarised into one sum $\left[\Sigma_{\text {decile 1-decile 10 }}\right.$ (CNI weights) $]$.

(4) These two sums were divided, so that the sum [ $\Sigma_{\text {decile 1-decile 10 }}$ $(\mathrm{CNI}$ weights $\times \mathrm{N})]$ was divided by the sum $\left[\sum_{\text {decile } 1 \text {-decile } 10}(\mathrm{CNI}\right.$ weights)]. The value thus obtained is called the final CNI weight and was calculated in the same way for each administrative area.

(5) The sum of the six final CNI weights was calculated $\left[\Sigma_{\text {area 1-area } 6}(\right.$ final CNI weights $\left.)\right]$.

(6) The allocation of resources to each administrative area in percentage is obtained by dividing the final CNI weight for each administrative area by the sum of the final CNI weights ( $\Sigma_{\text {area 1-area } 6}$ (final CNI weights) for all administrative areas (multiplied by 100). The proposed CNI model was compared with the official model used in Stockholm County.

\section{Statistical method}

The prevalence of poor self reported health status was standardised by sex and age (indirect standardisation). ${ }^{12}$ Hierarchical logistic regression was used in the analysis of data and the models were fitted using the SAS macro GLIMMIX. ${ }^{13}{ }^{14} \mathrm{~A}$ restricted maximum likelihood procedure was used in the method of estimation. The inclusion of a macro error term mixed the model with the CNI as a random effect. The interactions between fixed effect factors and the CNI were treated as random effects. No such interactions were found. The results were exhibited as odds ratios (OR) with 95\% confidence intervals (CI). The fit of the model was inferred from an extra-dispersion parameter, which met this demand.

The reliability of the items included in SALLS was analysed by means of re-interviewing (test-retest method), giving $\kappa$ coefficients between 0.7 and $0.9 .^{15}$

\section{RESULTS}

Poor self reported health in CNI deciles in a random sample of the Swedish population

Table 2 shows the odds ratio (OR) with the 95\% confidence intervals (CI) and the prevalence of poor self reported health status in different CNI deciles in a hierarchical logistic model adjusted for sex and age (25-74 years). Each transformed CNI decile includes about 2600 people representing a population of about 500000 .

The prevalence of poor self reported health increased significantly with every CNI decile compared with the reference level (decile 1) with the exception of decile 2, indicating an increasing prevalence of poor self reported health from $18 \%$ in the first most affluent decile to $33.8 \%$ in the tenth most deprived decile by increasing social deprivation. The prevalence of poor self reported health was about equal (22.8 to $24.9 \%)$ in deciles $4-7$. 
Table 4 The population of Stockholm County by administrative area and CNI decile. The number and percentage(in parentheses) of people in different deciles and administrative areas are shown

\begin{tabular}{|c|c|c|c|c|c|c|c|}
\hline \multirow[b]{2}{*}{ CNI deciles } & \multicolumn{7}{|c|}{ Administrative area } \\
\hline & NE & NW & NS & SS & SW & SE & Total \\
\hline $1(60.9-<81.6)$ & $56277(23)$ & $30234(10)$ & $43412(12)$ & $3469(1)$ & $17761(5)$ & $24083(10)$ & 175236 \\
\hline $2(81.6-<86.4)$ & 45236 (18) & 26280 (9) & $20101(5)$ & $14273(6)$ & 37437 (10) & 30844 (13) & 174171 \\
\hline $3(86.4-<90.9)$ & 32891 (13) & 43508 (15) & $18593(5)$ & 9447 (4) & $34729(10)$ & $36322(16)$ & 175490 \\
\hline $4(90.9-<95.0)$ & 30665 (13) & $10604(4)$ & $66696(18)$ & 8346 (3) & $34360(10)$ & 24502 (11) & 175173 \\
\hline $5(95.0-<98.3)$ & $29960(12)$ & $15296(5)$ & $75323(20)$ & $27392(11)$ & $14229(4)$ & $15438(7)$ & 177638 \\
\hline $6(98.5-<102.1)$ & $14970(6)$ & $35951(12)$ & $39699(11)$ & $38758(15)$ & $20791(6)$ & 21507 (9) & 171676 \\
\hline $7(102.1-<106.8)$ & $16381(7)$ & $35582(12)$ & $17247(5)$ & $62904(25)$ & $33894(9)$ & $7710(3)$ & 173718 \\
\hline $8(106.8-<112.9)$ & 9047 (4) & 34731 (12) & $30178(8)$ & 34197 (14) & $49228(14)$ & 19101 (8) & 176482 \\
\hline 9 (1 12.9-<120.6) & 9225 (4) & 32612 (11) & $12321(3)$ & 39895 (16) & 50875 (14) & $32063(14)$ & 176991 \\
\hline $10(\geqslant 120.6)$ & $0(0)$ & $25114(9)$ & $48946(13)$ & $13239(5)$ & $64116(18)$ & $18142(8)$ & 169557 \\
\hline Totals, Distribution, different areas & $244652(14)$ & $289912(17)$ & $372516(21)$ & 251920 (14) & $357420(20)$ & $229712(13)$ & $1746132(100)$ \\
\hline
\end{tabular}

Table 5 A comparison of indices for the allocation of economic resources to primary health care (percentage), CNI, and percentage of the variables included in the $\mathrm{CNI}$ and low income in the six different administrative areas of Stockholm County

\begin{tabular}{|c|c|c|c|c|c|c|c|}
\hline \multirow{2}{*}{$\begin{array}{l}\text { Indices and percentage immigrants and people with low income or } \\
\text { education }\end{array}$} & \multicolumn{7}{|c|}{ Administrative area } \\
\hline & NE & NW & NS & SS & SW & SE & Total \% \\
\hline Distribution by $\mathrm{CNI}$ model & 12.7 & 16.6 & 21.2 & 15.1 & 21.4 & 12.9 & 100.0 \\
\hline Distribution by official model (consumption-need model) & 12.4 & 16.4 & 22.0 & 16.8 & 20.2 & 12.2 & 100.0 \\
\hline $\mathrm{CNI}$ mean (transformed) & 90 & 97 & 97 & 102 & 101 & 95 & \\
\hline $\begin{array}{l}\text { Immigrants from Finland, Southern and Eastern Europe, Asia, Africa } \\
\text { and South America }\end{array}$ & 7.8 & 18.0 & 22.7 & 12.3 & 28.0 & 11.3 & 100.0 \\
\hline Low educational status ( $<10$ years) & 11.2 & 17.5 & 18.3 & 13.1 & 25.6 & 14.3 & 100.0 \\
\hline Elderly persons living alone & 12.1 & 14.1 & 26.3 & 20.8 & 18.1 & 8.7 & 100.0 \\
\hline Single parents & 11.7 & 17.3 & 18.9 & 16.0 & 21.8 & 14.4 & 100.0 \\
\hline Unemployed people & 9.3 & 16.0 & 21.6 & 15.9 & 24.7 & 12.5 & 100.0 \\
\hline High mobility & 11.8 & 17.8 & 22.1 & 15.2 & 20.8 & 12.3 & 100.0 \\
\hline Children under age 5 & 13.9 & 17.2 & 19.4 & 12.9 & 21.9 & 14.7 & 100.0 \\
\hline Low income (the lowest quartile) & 15.8 & 14.2 & 22.3 & 18.2 & 19.4 & 10.0 & 100.0 \\
\hline
\end{tabular}

There was a significant strong association between the transformed CNI and poor self reported health (table 2). There was a clear gradient, an approximately $150 \%$ increased risk of poor self reported health status for people living in the most deprived neighbourhoods $(\mathrm{OR}=2.50)$ compared with those living in the most affluent neighbourhoods $(\mathrm{OR}=1)$. The $\mathrm{OR}$ of poor self reported health status was about 1.5 in deciles 4 to 7 , representing 2 million of the Swedish population. Respondents living in neighbourhoods with addresses that could not be matched with SAMS areas had an increased risk of poor self reported health status with OR=1.51 (95\% CI; 1.25 to $1.81)$.

\section{CNI weights in corresponding deciles in Stockholm County}

Table 3 shows the mean CNI weights and CNI ratios in deciles for Sweden $(n=8.5$ million inhabitants) and Stockholm County $(\mathrm{n}=1.7$ million inhabitants). The ratio between the tenth and the first decile was 1.70 for Sweden and 1.66 for Stockholm—-that is, the CNI ratios were almost identical.

\section{Comparison between CNI deciles in Stockholm County and poor self reported health}

There was a strong association (hierarchical logistic regression) between the transformed CNI and poor self reported health in table 2, based on a random sample $(n=27346)$ of the total population of Sweden. In table 3 it was shown that the CNI ratios increased with increasing CNI deciles (increasing deprivation) based both on the population of Sweden and the population of Stockholm County. As they were very similar, it is concluded that the strong association between the transformed CNI and poor self reported health obtained in a random sample of the Swedish population also exists in Stockholm County.

\section{Application of $\mathrm{CNI}$ as an allocation resource index in Stockholm County}

The population of Stockholm County with the number and percentage of persons by administrative area and CNI decile is presented in table 4, which shows that the demographic structure differs in the administrative areas.

In table 5, the transformed CNI model was compared with the official consumption and need model currently used for the allocation of economic resources (percentages) to the primary health care services in Stockholm County. To evaluate the neighbourhood social position, information about the area $\mathrm{CNI}$, the proportion of immigrants, people with low educational status, low income, elderly people living alone, single parents, unemployed people, high mobility and children under age 5 is shown in table 5. CNI means for the six different areas have been calculated and show that some of the areas can be regarded as being more deprived. None of the most deprived neighbourhoods (decile 10) were found in the affluent north eastern (NE) part of Stockholm. The largest differences in allocation of resources when the CNI model was used were seen in the SS and SW areas. The SS area, which was as deprived as the SW area according to CNI, received $1.7 \%$ less resources with the CNI model than with the official model and the SW area received $1.2 \%$ more resources when the CNI model was used. The difference $21.4 \%$ compared with $20.2 \%$ 


\section{Key points}

- Care Need Index might be used as a direct empirica measure for a total distribution of resources to primary health care.

- $\mathrm{CNI}$ ratios corresponded to ORs for poor self reported health when the population in Stockholm County was divided into deciles.

- The CNI model, which is based on measures of material and social need, constitutes a better theoretical basis for the distribution of primary health care resources than the consumption of primary health care in previous years.

seems slight, but as the total annual budget for primary health care in Stockholm is 3.3 billion kronor, these additional resources would result in more than 50 new GPs for the deprived SW area. However, the SS area loses the same amount of economic resources. Therefore, even small differences in the allocation of resources, if calculated in percentages, are of great importance and represent considerable amounts of money. There were not so large changes in the allocation of resources in the NS and SE areas, $-0.8 \%$ and $+0.7 \%$, respectively. In the other two areas (NE and NW) in the northern part of Stockholm, there were only minor changes when the CNI model was used.

\section{DISCUSSION}

One of the main findings of this study was that CNI, after the transformation to a positive scale, might be used as a direct empirical measure for a total distribution of resources to primary health care. Moreover, the transformed CNI ratios corresponded to ORs for poor self reported health when the population in Stockholm County was divided into deciles. In this study, we have developed a need based model for the distribution of primary health care resources, an alternative to the official consumption model. The CNI model reallocated resources to a large extent, compared with the official consumption model, in the two most deprived administrative areas, the SS and the SW area. The SW area, with a $40 \%$ larger population than the SS area, received more resources while the SS area received less. Although it is not possible to conclude which model is the best for allocating resources to primary health care, the CNI model has a better theoretical approach - that is, measures of social need, rather than actual consumption. It may be possible that the consumption model reflects the actual access to health care so that an area with a high access to primary health care has a higher consumption of medical services. Therefore, we argue that the consumption model preserves established behaviour instead of measuring people's need for medical services.

An important concern is the significance and interpretation of the outcome factor. For example, qualms about the significance of self rated health status have been suggested by an Australian study that found unexpected gender differences in health status. ${ }^{16}$ In contrast, a Finnish longitudinal survey demonstrated the stability over time of self reported health status and that such a subjective health assessment was a valid indicator of health in middle aged populations. ${ }^{17}$ According to a study from Quebec, self reported health is the best indicator of population morbidity at the primary care level "since it tends to reflect individuals' perceptions of their health in the context of their expectations for their health". ${ }^{18}$ Self reported health status is widely used in European ${ }^{19-21}$ and in American ${ }^{22-25}$ studies as a useful predictor of the health conditions of a population, but it is possibly a subjective and imprecise measure of health, which reflects a person's general perception of the quality of life. However, the test-retest reliability of self rated health status was good when performed in another population from SALLS $1989 .{ }^{26}$
The finding that the CNI ratios and the odds ratios of poor self reported health showed a clear gradient, increasing with each decile with increasing deprivation, agreed with studies from the UK in particular. For example, people living in deprived neighbourhoods in London had an increased risk of poor self reported health, ${ }^{27}$ a finding in agreement with the present study. Moreover, the primary health care consultation rates were higher in the deprived neighbourhoods than in the affluent neighbourhoods. ${ }^{27}$ Thus, high rates of poor self reported health in deprived areas may be interpreted as a high need for primary health care. In another study from the UK, the authors showed that socioeconomic and demographic factors such as unemployment, living in a rented accommodation, and being from the Indian subcontinent were associated with more frequent consultations with GPs, even after adjustment for individual social position. ${ }^{28}$ In contrast, a British study using the Jarman score or the Townsend score did not reveal any association between these scores and poor self reported health. ${ }^{29}$

There is also an association between deprivation and other health outcomes. For example, people living in the deprived neighbourhoods in Sweden, as measured by the CNI, have higher rates of psychiatric hospital admissions, alcohol clinic admissions, cardiovascular risk factors (smoking, no physical activity, obesity), all cause mortality and coronary heart disease mortality. ${ }^{530}$ The pressure on primary health care centres located in such areas is therefore considerable. ${ }^{727}$ Although a causal association between social deprivation and health is difficult to prove, surveys in the UK and Sweden revealed that many GPs regarded socioeconomic factors such as a high proportion of unemployment, elderly people living alone, and single mothers as having a direct effect on their workload. ${ }^{131}$

The CNI is based exclusively on the need for health care, in contrast with the official pragmatic model used in Stockholm County, which is a combined model based on need and the previous year's consumption of primary health care. This is also the traditional way health authorities in the UK calculate the budget for primary health care, a budget based on historical expenditure, with an allowance for deprivation. In a study from Scotland, it was suggested that progressivity in the delivery of health care could be regarded as a frontier problem analogous to efficiency. ${ }^{32}$ In Sweden, as in several other Western countries, recruitment of GPs to the most deprived neighbourhoods has been difficult. A more progressive distribution of resources to the primary health care centres in the most deprived neighbourhoods would facilitate the recruitment of GPs so that, hopefully, people's health in these areas would also improve. It should be pointed out that, in this study, the tenth decile showed a much higher risk of poor self reported health $(\mathrm{OR}=2.5)$ than the corresponding CNI ratio (1.7). This indicates that, after the total distribution of resources has been made, additional resources may be provided for the tenth, most deprived decile.

This study has a few limitations as it is based on cross sectional data that do not permit inferences to be drawn about plausible pathways. Because of non-response, the prevalence of poor self reported health in the population may be underestimated to some extent, although probably not the relative measures. About 5\% of all respondents could not be matched with CNI scores, possibly because of incorrect addresses or new residential areas without CNI scores. To gain power, these missing data were not excluded from the analysis. The risk of this introducing a bias is probably small because the risk would be at the same level in the whole Swedish population. The transformed CNI is currently focused on Stockholm, a big city with 1.7 million inhabitants, which might pose problems of generalisability. However, our findings agree with other studies from $\mathrm{UK},{ }^{27}{ }^{28}$ and therefore we believe that they have wider application. In other developed countries CNI could be adapted as a practical tool to facilitate a total distribution of 


\section{Policy implications}

Care Need Index may be used as a basis for political decision makers re-distributing economic resources to primary health care. Additional economic resources to GP incentives in deprived neighbourhoods, where the need is greatest, would make it possible to increase the number of GPs and district nurses and improve diagnostic procedures. Although we still cannot provide evidence that the $\mathrm{CNI}$ model actually redistributes more resources to the most deprived neighbourhoods than the official model does it is based on measures of material, social and cultural needs, which constitute a better theoretical approach than the consumption model. However, redistribution of additional resources to deprived neighbourhoods should be done with care because of the limited economic resources in the total health care budget.

primary health care resources based on need. The analysis of the association between the transformed CNI and poor self reported health is based on a random sample of the Swedish population, but the allocation of resources is restricted to Stockholm County and constitutes a limitation. However, the conclusions concerning the association between the transformed CNI and poor self reported health obtained for the whole population of Sweden can be generalised to Stockholm County because of the close similarity between the CNI ratios in Sweden and Stockholm County, which were almost identical.

Furthermore, this study also has some additional strengths. The use of a multilevel technique provides information at both the individual and the neighbourhood level. Another substantial strength of the study is that it is based on a large and well defined simple random sample of the Swedish population. Finally, it is easy to update the index because the items in CNI are renewed annually.

\section{Recommendations}

CNI may be used as a basis for political decision makers re-distributing economic resources to primary health care. Additional economic resources to GP incentives in deprived neighbourhoods, where the need is greatest, would make it possible to increase the number of GPs and district nurses and improve diagnostic procedures. Although we still cannot provide evidence that the CNI model actually redistributes more resources to the most deprived neighbourhoods than the official model does, it is based on measures of material and social needs, which constitute a better theoretical approach than the consumption of primary health care in previous years. However, re-distributing of additional resources to deprived neighbourhoods should be done with care because of limited economic resources in the total health care budget. Finally, the CNI can be used to distribute total primary health care resources.

\section{Authors' affiliations}

K Sundquist, M Malmström, S-E Johansson, J Sundquist, Karolinska Institutet, Family Medicine, Stockholm, Sweden

Funding: this study was supported by grants from the Swedish Medical Research Council (KO1-27X-1 1651-04A), the Swedish Council for Social Research, the Stockholm County, the Axel and Margaret Johnson Foundation, National Institutes of Health (1RO1 HL71084-01), and the Knut and Alice Wallenberg Foundation to Jan Sundquist.

Conflicts of interest: none.

\section{REFERENCES}

1 Malmström M, Sundquist J, Bajekal M, et al. Indices of need and social deprivation for primary health care. Scand J Soc Med 1998;26:124-30.
2 Bajekal M, Alves B, Jarman B, et al. Rationale for the new GP deprivation payment scheme in England: effects of moving from electora ward to enumeration district underprivileged area scores. Br J Gen Pract 2001:51:451-5

3 Dalgard O. Bomiliø og psykisk helse [Environment and mental health]. [Dissertation] [In Norwegian with English summary]. Oslo: University of Oslo, 1980

4 Cullberg J, Stefansson CG, Wennersten E. Psychiatry in low status dwelling areas. Psychiatr Soc Sci 1981;1:117-23.

5 Malmstrom $M$, Sundquist J, Johansson SE, et al. The influence of social deprivation as measured by the $\mathrm{CNI}$ on psychiatric admissions. Scand J Public Health 1999;27: 189-95.

6 Andren KG, Rosenqvist U. An ecological study of the relationship between risk indicators for social disintegration and use of a somatic emergency department. Soc Sci Med 1987;25:1121-7.

7 Sundquist J, Rosén U. The influence of social surroundings on consultation of private care, emergency department, hospital out-patient departments, and primary health care. 1384 Consultations made by a defined population in a residential area. Eur $J$ of Publ Health 1993;3:188-92.

8 Malmstrom $\mathbf{M}$, Sundquist J, Bajekal $M$, et al. Ten-year trends in all-cause mortality and coronary heart disease mortality in socio-economically diverse neighbourhoods. Public Health 1999;1 13:279-84.

9 Sundquist J. Ethnicity as a risk factor for mental illness. A population-based study of 338 Latin American refugees and 996 agesex- and education-matched Swedish controls. Acta Psychiatr Scand 1993;87:208-12

10 Andersson PA, Varde E, Diderichsen F. Modelling of resource allocation to health care authorities in Stockholm county. Health Care Manag Sci 2000:3:141-9.

11 Statistics Sweden. The Swedish survey of living conditions. Design and methods. Stockholm: Statistics Sweden, 1996:appendix 16

12 Breslow NE, Day NE. Statistical methods in cancer research. Vol 1. Lyon, France: International Agency for Research on Cancer, 1980.

13 Wong GY, Mason WM. Hierarchical logistic regression for multilevel analysis. J Am Stat Assoc 1985;80:513-24.

14 Littell RC, Millkien GA, Stroup WW, et al. SAS system for mixed models. Cary, NC: SAS Institute, 1996.

15 Wärneryd B. Levnadsförhållanden. Återintervjustudie i undersökningen av levnadsförhållanden 1989 (ULF). [Living conditions. Reinterview in ULF 1989.] Stockholm: Statistics Sweden, 1991:appendix 12.

16 McCallum J, Shadbolt B, Wang D. Self-rated health and survival: a 7-year follow-up study of Australian elderly. Am J Public Health 1994;84:1 100-5

17 Miilunpalo S, Vuori I, Oja P, et al. Self-rated health status as a health measure: the predicitive value of self-reported health status on the use of physician services and on mortality in the working-age population. J Clin Epidemiol 1997;50:517-28

18 Birch S, Eyles J, Newbold KB. Proxies for healthcare need among populations: validation of alternatives - a study in Quebec. J Epidemiol Community Health 1996;50:564-9.

19 Power C, Matthews S, Manor O. Inequalities in self-rated health: explanations from different stages of life. Lancet 1998;351:1009-14.

20 Krause NM, Jay GM. What do global self-rated health items measure? Med Care 1994;32:930-42.

21 Fernández de la Hoz K, Leon DA. Self-pereived health status and inequalities in use of health services in Spain. Int J Epidemiol 1996:25:593-603.

22 Kaplan GA, Camacho T. Perceived health and mortality: a nine-year follow-up of the human population laboratory cohort. Am J Epidemiol 1983; 117:292-304.

23 Idler EL, Angel RJ. Self-rated health and mortality in the NHANES-I Epidemiologic Follow-up Study. Am J Public Health 1990;80:446-52.

24 Grant MD, Piotrowski ZH, Chappell R. Self-reported health and survival in the Longitudinal Study of Aging, 1984-1986. J Clin Epidemiol 1995:48:375-87.

25 McGee DL, Liao Y, Cao G, et al. Self-reported health status and mortality in a multiethnic US cohort. Am J Epidemiol 1999;149:41-6.

26 Lundberg 0 , Manderbacka K. Assessing reliability of a measure of self-rated health. Scand J Soc Med 1996;24:218-24.

27 Curtis SE. Use of survey data and small area statistics to assess the link between individual morbidity and neighbourhood deprivation. $J$ Epidemiol Community Health 1990;44:62-8.

28 Carr-Hill RA, Rice N, Roland M. Socioeconomic determinants of rates of consultation in general practice based on fourth national morbidity survey of general practice. BM 1996:312:1008-13.

29 Jessop EG. Individual morbidity and neighbourhood deprivation in a non- metropolitan area. J Epidemiol Community Health 1992;46:543-6.

30 Sundquist J, Malmstrom M, Johansson SE. Cardiovascular risk factors and the neighbourhood environment: a multilevel analysis. Int J Epidemiol 1999;28:841-5.

31 Jarman B. Identification of underprivileged areas. BM 1983;286:1705-9

32 Sutton $\mathbf{M}$, Lock $P$. Regional differences in health care delivery: implications for a national resource allocation formula. Health Econ 2000;9:547-59. 